\title{
Dados à mesa: visualizando a alimentação na cidade de São Paulo
}

\author{
Feeding data: visualizing São Paulo City food system
}

Gabriela Momberg Araujo, Leandro Manuel Reis Velloso

infovis, alimentação, estamparia

O artigo apresenta e discute resultados de um trabalho final de graduação que teve como principal objetivo explorar a visualização de dados relacionados ao sistema alimentar, integrando metodologias do Design da Informação, da Design Research e do Design Centrado no Humano. O projeto Dados à mesa teve como resultados, expressos por meio da estamparia de uma coleção têxtil, diferentes perspectivas sobre a alimentação e sua espacialidade na cidade de São Paulo. A elaboração de um projeto de design para comunicação de dados de interesse público mobilizou um percurso de pesquisa e experimentação caracterizado pela interdisciplinaridade.

infovis, food system, stamping

This article presents and discusses some of the results from a final degree project whose main goal was to explore the visualization of food system data, combining Information Design, Design Research and Human Centered Design. The project, called Feeding data, has resulted in a stamping collection that brings up different perspectives on the food system and its spatial distribution in the city of São Paulo. Elaborating a design project for the communication of public interest data led to an interdisciplinary path of research and experimentation.

\section{Introdução}

A visualização de dados é uma das áreas do design da informação por meio da qual se busca permitir análises, exploração e descobertas (Cairo, 2016). Embora seja tradicionalmente utilizada como ferramenta de apoio nos meios científico e acadêmico, importantes desdobramentos da revolução tecnológica abriram o caminho para investigações conceituais que evidenciam o papel do design na construção de visualizações (Viégas \& Wattenberg, 2007). O emprego de técnicas de infovis pode servir para dar transparência a questões de interesse público, funcionando como chamamento à participação social (Schoffelen et al, 2015). Nosso trabalho se fundamenta na teoria de que a visualização de dados deve ser dirigida por evidências quantitativas, seguindo critérios científicos, que resultam em conhecimentos claros e precisos (Tufte, 1997, p. 53). Tais conhecimentos devem não apenas gerar a reflexão, mas também mover ações políticas e apoiar a tomada de decisão. Curitiba | Brazil | 2021 
Diante desse cenário, o projeto Dados à mesa: visualização de sistemas alimentares no território, fruto de Trabalho Final de Graduação apresentado em 2020 na Faculdade de Arquitetura e Urbanismo da Universidade de São Paulo, buscou explorar a infovis como forma de comunicar questões públicas que permeiam a alimentação no território paulistano. A partir de indicadores relacionados aos distritos administrativos do município, procuramos ilustrar as relações entre o espaço urbano e os diversos aspectos do sistema alimentar, como oferta, disponibilidade, consumo, trabalho, segurança e soberania alimentar. Camadas de informação social e econômica foram adicionadas às visualizações como forma de contextualização, permitindo a visão do tema sob diferentes perspectivas.

\section{Materiais e métodos}

O percurso deste projeto combinou metodologias da Design Research, do Design da Informação e do Design Centrado no Humano (DCH). A sequência de etapas que definiram o desenho da pesquisa pode ser vista na Figura 1.

A pesquisa inicial envolveu um levantamento bibliográfico, sobre dados e indicadores relacionados ao sistema alimentar e sua relação com o espaço urbano em São Paulo, bem como uma atividade de imersão no universo do usuário, abordagem fundamentada no DCH (Gould \& Lewis, 1985). Por meio de entrevistas semiestruturadas com pessoas conectadas de diferentes formas com a cadeia da alimentação, buscamos compreender as percepções em relação à espacialidade das práticas alimentares.

Uma análise exploratória dos dados levantados possibilitou a familiarização com a natureza dos mesmos (Kirk, 2016, p. 121), constituindo um conhecimento que foi incorporado na elaboração das visualizações. Para processar e analisar o resultado das entrevistas, utilizamos o método do Diagrama de Afinidades (Hanington, 2012), a partir do qual foram gerados os requisitos de projeto.

A ideação foi caracterizada pela geração de alternativas a partir da prática do sketching. O desenho manual, como apontado por Lupi (2017) contribui para a reflexão sobre a essência dos dados, levando ao aperfeiçoamento progressivo da representação de forma consciente por parte do designer. Esta forma de experimentação visual foi de suma importância para o processo criativo, permitindo um exercício iterativo de geração e interpretação de ideias (Van der Lugt, 2002).

Os dados utilizados para a constituição dos produtos analisados neste artigo são provenientes do Mapeamento de Desertos Alimentares ${ }^{1}$ e do Índice Paulista de Vulnerabilidade Social ${ }^{2}$.

\footnotetext{
${ }^{1}$ https://aplicacoes.mds.gov.br/sagirmps/portal-san/artigo.php?link=23

2 http://ipvs.seade.gov.br/view/index.php
} 
Figura 1. Diagrama representativo da sequência de etapas da pesquisa.

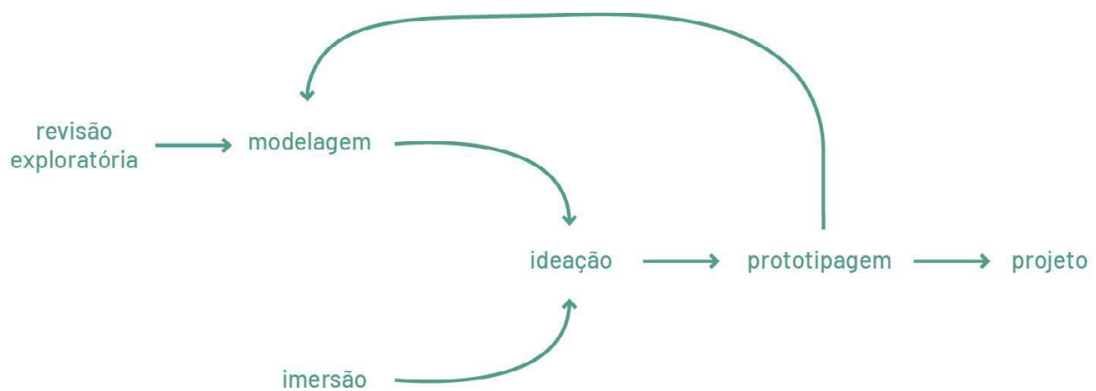

\section{Resultados}

\section{Resumo da solução}

O trabalho Dados à Mesa teve como produtos três coleções, cada uma delas identificada por uma cor secundária, compostas por panos de prato e guardanapos estampados (Figura 2). O presente artigo se concentra sobre o conjunto de guardanapos de uma das coleções propostas (Coleção 2). Os dados representados neste conjunto são relativos a alimentação e vulnerabilidade social.

Figura 2. Diagrama esquemático de arquitetura do conteúdo.
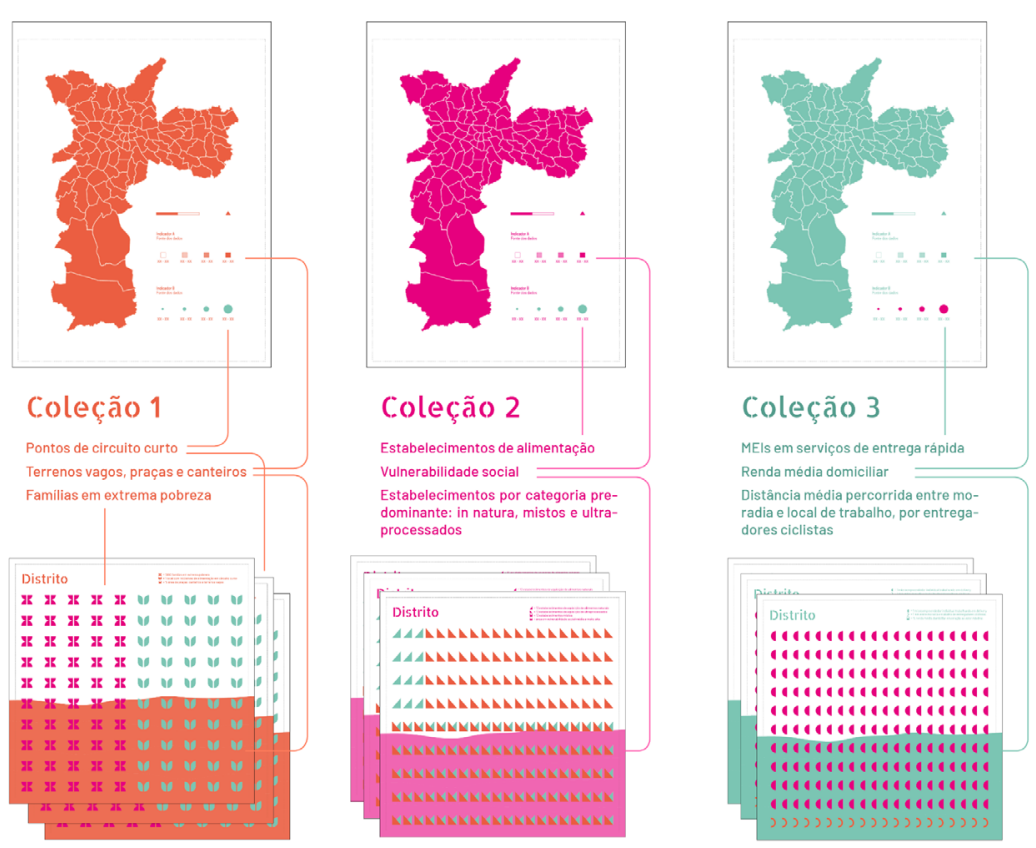


\section{Composição visual}

Cada guardanapo é a representação de uma unidade espacial, portanto foram geradas 96 variações de estampa - volume correspondente ao total de distritos paulistanos (Figura 3). 0 sistema de representação definido contempla informações quantitativas e categóricas: proporção de área em vulnerabilidade social, número de estabelecimentos e categoria de varejo a qual pertencem. A partir da observação de valores mínimos e máximos, de outliers e de proporções entre categorias, foi criada uma matriz comum visando a produção gráfica por stencil. A variação das estampas foi dada pelo preenchimento de determinada quantidade de pontos e pelo tingimento proporcional do tecido, conforme os dados do distrito representado.

Figura 3. Visão geral das visualizações geradas para os 96 distritos.

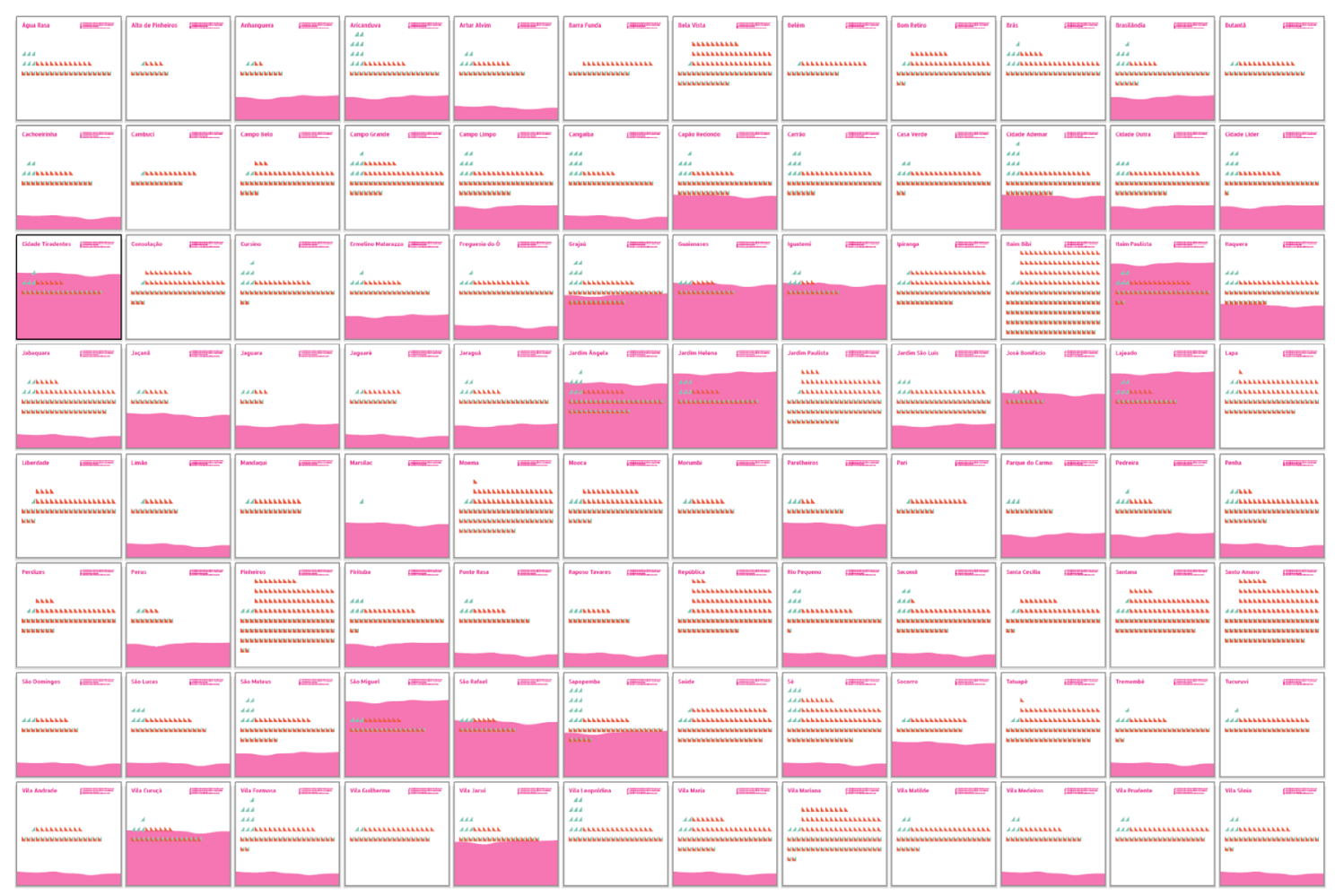

\section{Gráfico de símbolos}

Para representar a quantidade de estabelecimentos de alimentação, foram utilizados gráficos de símbolos (Figura 4), com cada ponto equivalente a dez estabelecimentos. O objetivo desta visualização era chegar o mais próximo possível dos números absolutos, representando em detalhe a magnitude das variações. Os pontos foram caracterizados por meio da variação de formas e de cores, como método de diferenciação de categorias. Seguindo essa diretriz, estabelecimentos de aquisição de ultraprocessados aparecem como triângulos em laranja, estabelecimentos de aquisição de alimentos in natura aparecem como triângulos verdes, em orientação oposta aos ultraprocessados. A sobreposição destas duas formas de orientações opostas e cores contrastantes representa a categoria de estabelecimentos mistos. 
Figura 4. Evolução do desenho do gráfico de símbolos, baseada nos valores máximos e mínimos de cada categoria.
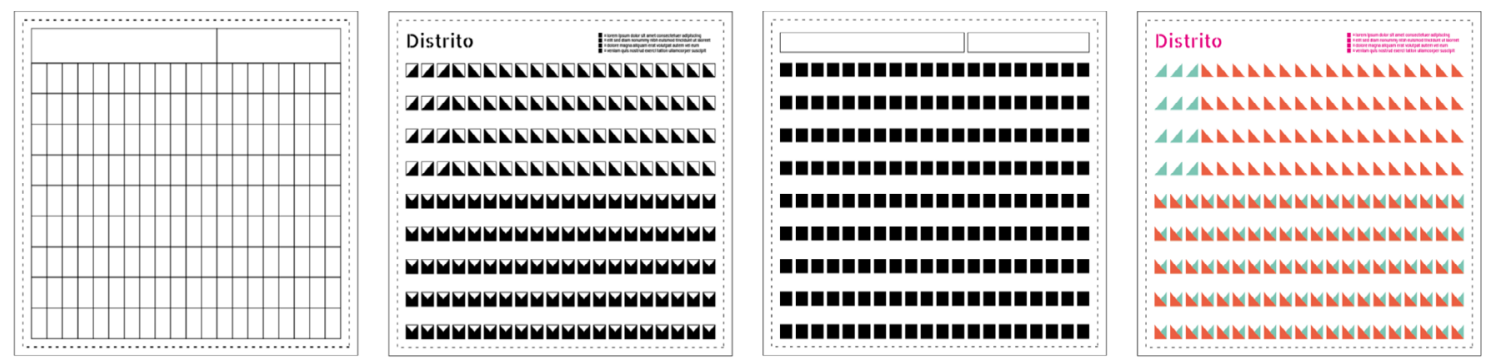

\section{Preenchimento de área}

Os dados de vulnerabilidade social foram representados por preenchimento de cor como plano de fundo da composição. A construção desta camada de informação consiste no preenchimento proporcional à área de vulnerabilidade média a alta no distrito, considerando a área total do guardanapo como a área total do distrito (Figura 5). A prototipação foi realizada a partir de imersão do tecido em tinta diluída, em profundidade determinada pelo dado.

O efeito visual ocasionado pela presença da cor permite identificar os distritos em situação mais crítica no território, além de colocar a situação da oferta de alimento em perspectiva por meio desta contextualização social.

Figura 5. Evolução do design do gráfico de preenchimento de área com base em proporções de imersão.
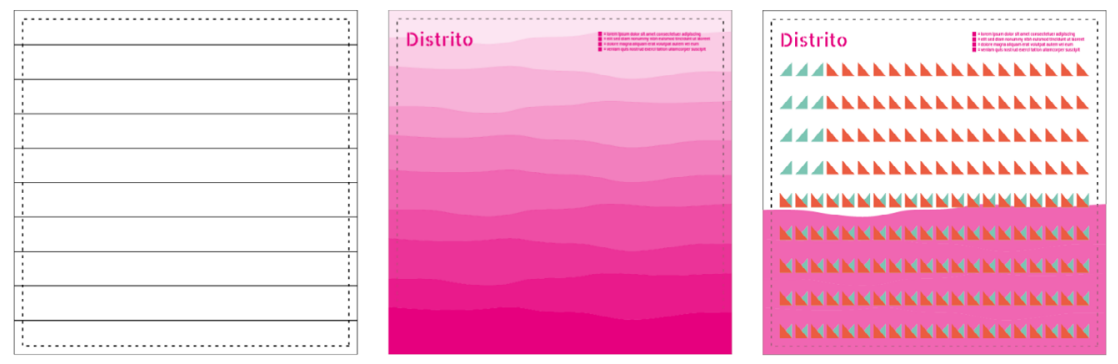

\section{Discussão}

\section{Arquitetura da informação}

Parte da análise sobre os resultados pode ser feita sob a luz da arquitetura da informação, visto que se organizam em diferentes escalas e níveis de abstração. A visualização do território a partir da repetição de unidades representativas de suas subdivisões, possibilitou diversas abordagens de leitura. Por meio da interação com os objetos há uma alternância de recortes e escalas de análise. Quando visualizado individualmente, o artefato remete à escala local, podendo estabelecer um diálogo direto com o usuário, cujo objetivo é suscitar a curiosidade sobre o ambiente em seu entorno. Ao comparar duas ou mais visualizações, pode-se navegar pelas diferenças e semelhanças entre distritos, esperamos com isso provocar a reflexão e o questionamento no leitor. Em um nível de abstração mais amplo, observa-se no conjunto um 
cartograma que ilustra a variação dos dados ao longo do território de São Paulo, que pode ser organizado livremente pelo observador.

\section{Suporte}

Vale destacar que suporte material configura não apenas a experiência sensorial, mas também sugere uma ambientação da leitura. Ao utilizar os guardanapos como suporte para as visualizações, o projeto abre espaço para uma leitura em meio à prática alimentar mais cotidiana: o consumo (Figura 6). Nesse sentido, a intenção do projeto é a de incentivar uma alteração da perspectiva para a reflexão sobre a alimentação como questão pública, utilizando como veículo uma composição que poderia ser definida como Casual Infovis, ou seja, integrada à rotina do usuário, proporcionando contemplação sobre o significado dos dados sem associá-los a uma operação específica a ser cumprida (Pousman et al, 2007).

Figura 6. Fotografia dos protótipos na ambientação proposta.

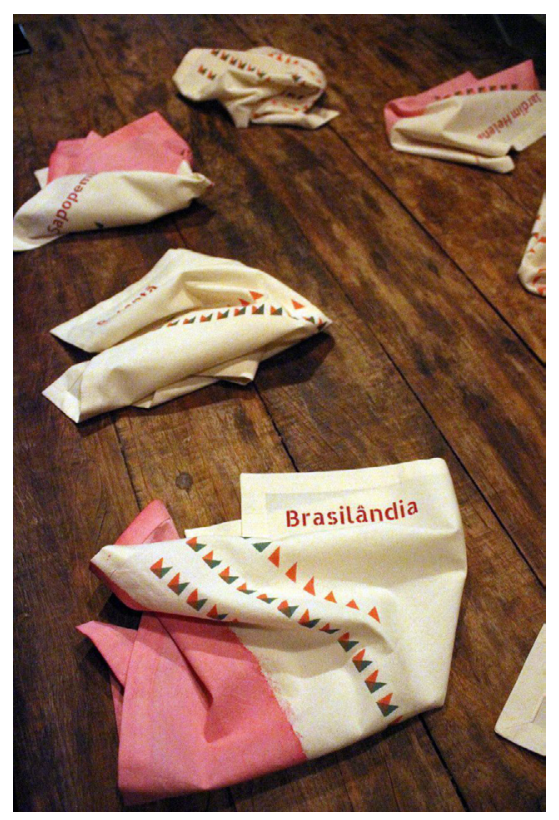

\section{Estrutura e repetição}

A criação de uma marca visual única para cada componente é um efeito relevante, o que todavia envolve algumas limitações. Diante do desafio de representar a totalidade do município, foi necessário incorporar o alto contraste que resulta de suas desigualdades. A repetição de uma mesma matriz sobre todas as unidades, baseada na representação de small multiples, serve ao propósito de análise comparativa em que se prioriza a variação de dados, em detrimento da variação de estrutura (Tufte, 1990, p. 67). Para ilustrar essa condição, basta perceber que alguns distritos proporcionam grandes vazios no respectivo tecido, não sendo o seu layout alterado em função disso (Figura 7). Assim, ainda que haja possibilidade de visualização isolada do distrito, é uma premissa que cada artefato faça sentido dentro do conjunto. 
Figura 7. Exemplos de variações da estampa.
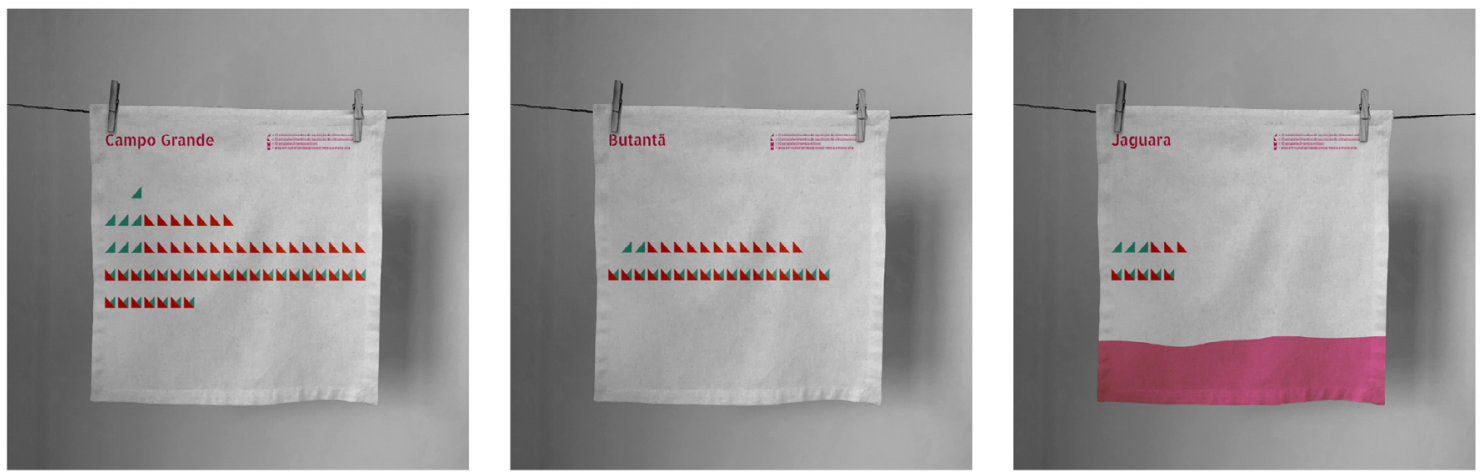

\section{Experimentação}

Tanto o processo quanto o resultado carregam reflexões sobre a visualização de dados. A confecção das peças, embora tenham sido modeladas digitalmente, envolveu uma experimentação gráfica intensa durante sua ideação por meio do sketching, além de um estudo experimental de técnicas de impressão para a prototipagem em tecido (Figura 8). Junto à dimensão visual e espacial destes produtos, destaca-se a dimensão temporal relacionada ao desenho manual e à estamparia artesanal, com uma duração diferente para cada combinação de dados. Isso contribui para o desenvolvimento de uma postura mais sensível por parte do designer em relação à realidade que busca representar (Lupi, 2017). Por outro lado, a interação do público final com as visualizações pode ocorrer de forma mais imediata ou mais prolongada, a depender de como o observador decide explorar detalhes e organizar elementos.

Figura 8. Fotografia do processo de produção gráfica por stencil.

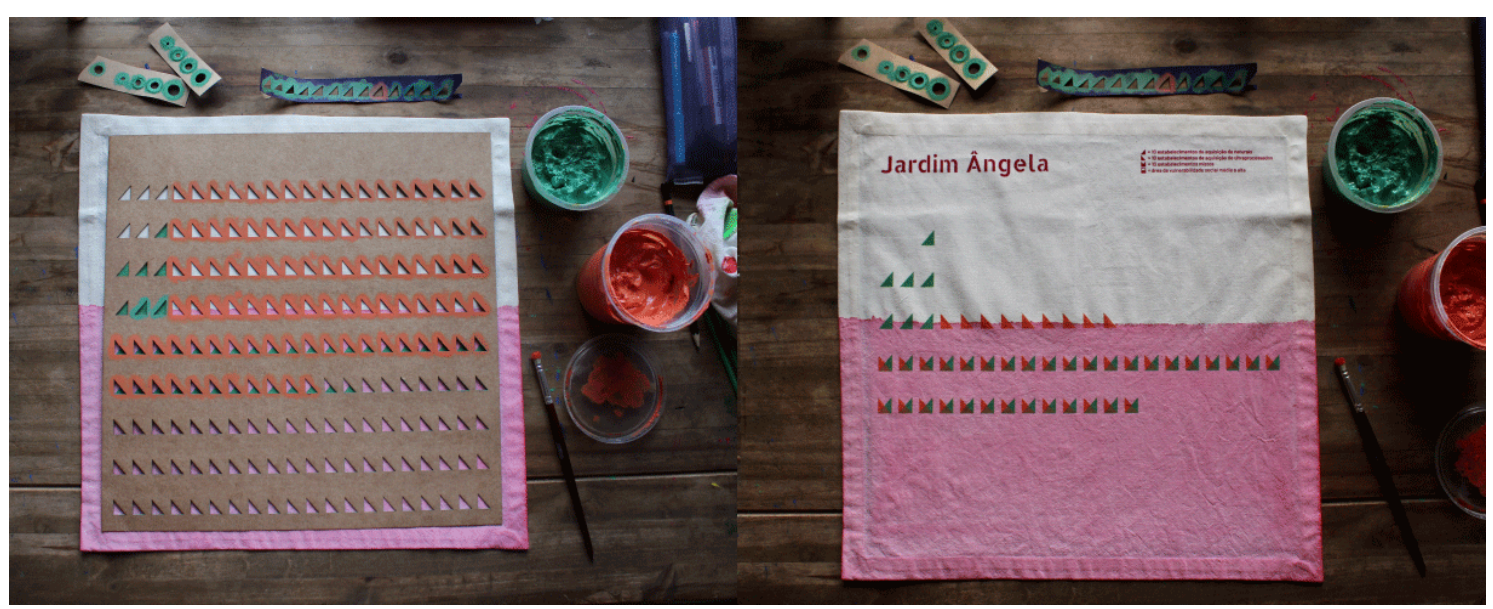

\section{Conclusão}

O resultado apresentado neste artigo constitui parte do Trabalho Final de Graduação em Arquitetura e Urbanismo intitulado Dados à mesa: visualização de sistemas alimentares no território. O trabalho se desenvolveu a partir da busca por formas de representação de questões complexas de interesse público, como é o caso da segurança alimentar. Foram 
investigadas metodologias diversas no esforço de transformar a visualização de dados abertos em comunicação voltada para o engajamento do público. Nesse sentido, foi fundamental entender diferentes perspectivas sobre o tema a partir de bibliografias interdisciplinares e da pesquisa de usuário, que subsidiaram o processo criativo de Infovis.

\section{Referências}

Cairo, A. (2016). The Truthful Art: Data, Charts and Maps for Communication. New Riders.

Gould, J. D., \& Lewis, C. (1985). Designing for usability: key principles and what designers think. Communications of the ACM. 28(3), 300-311. https://doi.org/10.1145/3166.3170

Hanington, B., \& Martin, B. (2012). Universal Methods of Design. Beverly: Rockport Publishers.

Kirk, A. (2016). Data visualisation: A handbook for data driven design. Sage.

Lupi, G. (2017). Data Humanism, the Revolution will be Visualized. Giorgia Lupi. Disponível em https://medium.com/@giorgialupi/data-humanism-the-revolution-will-be-visualized$31486 \mathrm{a} 30 \mathrm{dbfb}$

Pousman, Z., Stasko, J., \& Mateas, M. (2007). Casual Information Visualization: Depictions of Data in Everyday Life. IEEE Transactions on Visualization and Computer Graphics, 13(6), 1145-1152. https://doi.org/10.1109/TVCG.2007.70541

Schoffelen, J., Claes, S., Huybrechts, L., Martens, S., Chua, A., \& Moere, A. V. (2015). Visualising things. Perspectives on how to make things public through visualisation. CoDesign. 11(3-4), 179-192. https://doi.org/10.1080/15710882.2015.1081240

Tufte, E. (1990). Envisioning information. Cheshire: Graphic Press LLC.

Tufte, E. (1997). Visual Explanations: Images and Quantities, Evidence and Narrative. Connecticut: Graphic Press.

Van der Lugt, R. (2002). Brainsketching and How it Differs from Brainstorming. Creativity and Innovation Management, 11(1), 43-54. https://doi.org/10.1111/1467-8691.00235

Viégas, F., \& Wattenberg, M. (2007). Artistic Data Visualization: Beyond Visual Analytics. Schuler D. (eds) Online Communities and Social Computing. OCSC 2007. Lecture Notes in Computer Science, 4564. Berlin, Heidelberg: Springer. https://doi.org/10.1007/978-3-54073257-0_21

\section{Sobre o(a/s) autor(a/es)}

Gabriela Momberg Araujo, FAUUSP, Brasil <gabriela.momberg.araujo@alumni.usp.br> Leandro Manuel Reis Velloso, Dr, FAUUSP, Brasil <leandrovelloso@usp.br> 